\title{
Informal caregivers' attitudes and compliance towards a connected health platform for home care support: Insights from a long-term exposure
}

\author{
Estefanía Guisado-Fernández $M^{\mathrm{a}, *}$ \\ Guido Giunti MD PhD \\ Laura Mackey $\mathrm{PhD}^{\mathrm{c}}$ \\ Paula Alexandra Silva $\mathrm{PhD}^{\mathrm{d}}$ \\ Catherine Blake $\mathrm{PhD}^{\mathrm{c}}$ \\ Brian Caulfield $\mathrm{PhD}^{\mathrm{a}}$
}

\begin{abstract}
ansight Centre for Data Analytics, $3^{\text {rd }}$ floor O'Brien Science Building East, University College Dublin, Dublin, Ireland; ' ${ }^{\mathrm{M} 3 S}$ Research Unit, Faculty of Information Technology and Electrical Engineering, University of Oulu, Oulu, Finland; 'School of Public Health, Physiotherapy and Sports Science, University College Dublin, Dublin, Ireland; ${ }^{\mathrm{d} D e p a r t m e n t ~ o f ~ C o m p u t e r ~ S c i e n c e, ~ C e n t e r ~ f o r ~ I n f o r m a t i c s ~}$ and Systems of the University of Coimbra (CISUC), University of Coimbra, Coimbra, Portugal; *Corresponding author: estefaniaguisadofernandez@gmail.com
\end{abstract}

E. Guisado-Fernández, G. Giunti, L. Mackey, P.A. Silva, C. Blake, B. Caulfield. Informal caregivers' attitudes and compliance towards a connected health platform for home care support: Insights from a long-term exposure. Gerontechnology 2019;18(4):231-242; https://doi. org/10.4017/gt.2019.18.4.005.00 Background When designing Connected Health (CH) solutions for home care, it is vital to focus on usability and user experience to ensure that technologies are easy to use and meet users' expectations and needs. Generally, the usability and user experience tests are conducted during short-term exposure, which does not allow a true understanding of how the technology can help with the home caring tasks. Research aim We aim to investigate informal caregivers' feedback on the utility and usability of a $\mathrm{CH}$ monitoring platform for People with Dementia (PwD) during a period of extended use in the natural living context, and to understand how this was related to compliance patterns. Methods Informal caregiver's feedback about the $\mathrm{CH}$ platform, usability, and the impact of short-term versus long-term exposure were investigated through semi-structured individual interviews at the beginning and end of a 6-month deployment in the home care setting. Informal caregivers' compliance with the $\mathrm{CH}$ platform was analysed from their daily platform utilization during the deployment time. Results 11 informal caregivers agreed to participate. There was a change in the participants' opinions about the $\mathrm{CH}$ platform between the short-term and the long-term exposure feedback. Their initial impressions about what the $\mathrm{CH}$ platform could offer them to improve their delivery of home care for the PwD did not correspond with what they found that the $\mathrm{CH}$ platform could provide them following the long-term exposure. If at the beginning they saw the $\mathrm{CH}$ platform as a helpful tool to facilitate home care delivery and to improve their self-efficacy, after the deployment they expressed that because of the way the platform was designed it was mainly conceived for dementia research benefit and not to fulfill their caring needs. Compliance with the $\mathrm{CH}$ platform was quite low and similar between all participants. Conclusions Most contemporary $\mathrm{CH}$ studies are not conducted in real-life settings and without enough duration in time. Consequently, this is not providing accurate insights into the factual informal caregivers' interaction with these technologies and their suitability for their caring needs and support. With this study, we have recognized the importance of studying how informal caregivers' are engaging with $\mathrm{CH}$ for home care in their own environments and for enough duration of time. We have also highlighted that, despite the fact of applying $U C D$, the result may not always be satisfactory for the user. For these reasons, more longitudinal research on PwD and their informal caregivers, $\mathrm{CH}$ technologies adoption need to be conducted.

Keywords: Dementia, informal caregiver, connected health, usability, user experience 


\section{INTRODUCTION}

\section{Background}

Dementia is one of the most limiting chronic diseases with an estimated prevalence of 50 million people worldwide (World Health Organization, 2017). People with Dementia (PwD) are usually cared for at home by an 'informal caregiver', a family member or a close relative that takes care of the PwD in all of the aspects of their life, without any formal training or qualification, and without being paid for their work (Donelan et al., 2002). Being an informal caregiver can be a challenging and demanding role that can impact negatively on their own health, as the continuous burden and strain they suffer can lead them to experience some physical and mental health issues such as anxiety, depression, and stress (Godwin, Mills, Anderson, \& Kunik, 2013). These issues have important implications as they can jeopardize the ability of the informal caregiver to provide continuous care (Donelan et al., 2002).

Recent research suggests that technology-driven interventions, such as smartwatches, monitoring devices, or locator devices, can benefit home care delivery and consequently support the informal caregivers (Godwin et al., 2013). Technology can help the PwD to maintain independence, and improve their quality of life and health status (Boletsis, McCallum, \& Landmark, 2015) and at the same time it can provide the support the informal caregivers may need at home during this caring process (Bossen, Kim, Williams, Steinhoff, \& Strieker, 2015). With this, the literature advocates that technology applied to home care can offer potential opportunities to reduce the burden of informal caregiving and the need for premature nursing home placement, through a low-cost alternative for home care delivery and social support (Godwin et al., 2013).

This is the case for Connected Health $(\mathrm{CH})$, a new model of chronic care delivery facilitated by technology where all the stakeholders involved in a person's care are 'connected' through a health portal that provides a continuous and efficient flow of information between them (Caulfield \& Donnelly, 2013). The concept of $\mathrm{CH}$ has gained attention amongst Dementia researchers, as it has shown positive results in helping the informal caregivers in their delivery of home care for the elderly (Chouvarda, Goulis, Lambrinoudaki, \& Maglaveras, 2015; Darkins et al., 2008). Using a wide variety of technologies such as body-worn and monitoring devices, $\mathrm{CH}$ can help the informal caregiver in their caring duties, through the continuous monitoring of the health status of the PwD at home, alerting them to changes in the PwD and their environment (such as falls or any other emergency event), and facilitating their communication with the health- care professionals (HCP) when needed. $\mathrm{CH}$ also shows promise in reducing informal caregivers' burden, stress, anxiety, depression, and in bringing improvements to their quality of life (Godwin et al., 2013; Torkamani, 2014).

When designing $\mathrm{CH}$ solutions for home care, the literature suggests that it is necessary to include the final intended user (Guisado-Fernandez, Giunti, Mackey, Blake, \& Caulfield, 2019), in this case, the informal caregiver, during all the phases of the design, development, and implementation process (Johnson, Johnson, \& Zhang, 2005). This is important in order to ensure that the resulting technologies and devices fit into the informal caregivers' needs and meets their expectations for facilitating their caring work (Brown et al., 2014; Kort \& Van Hoof, 2014; Lundberg, 2014; Span et al., 2014). There are many examples in the literature of how informal caregivers' perceptions and feedback has been brought into the process of developing technology solutions to enable home care. These range from the informal caregivers being involved throughout the process from concept to final implementation and usability evaluation in the field (Hwang, Truong, \& Mihailidis, 2012; Lopes et al., 2016), to a model where PwD and their informal caregivers are only involved at the end of the process when their feedback is sought on a fully developed system (Cristancho-Lacroix et al., 2015; Pot, Blom, \& Willemse, 2015). Regardless of the extent of involvement across the implementation cycle, the user experience tests that are employed mainly take place in research environments (Feeney Mahoney, Coon, \& Lozano, 2016) and with a very limited duration of just a few hours (Hwang et al., 2012). Even in the case of home tests, their duration is equally restrained to just a few hours (Hattink, Droes, Sikkes, Oostra, \& Lemstra, 2016), or in the best cases for a limited number of weeks (Lewis, Hobday, \& Hepburn, 2010). This short-term exposure may not provide an opportunity for proper immersion into the real-life scenario, where an informal caregiver is involved in living with and using the technology for long-term exposure, over months as opposed to hours or weeks. During an extended period such as this, informal caregivers can develop a true understanding as to how the technology can either help or hinder them during their every day caring tasks. This enables a better perspective and ability to provide feedback on the technology and the service that it intends to enable. Therefore, there is a need to establish appropriate informal caregivers' evaluations that take place after long-term exposure to technology home care delivery interventions and placed in 'real world' scenarios (Bharucha et al., 2009). 
This paper describes a study investigating the effect of a long-term exposition on the perceived usability and user experience of a $\mathrm{CH}$ platform specifically created to provide home support for informal caregivers of PwD. The study presented here is nested inside the "Connected Health Supporting Home Stay in Dementia (CHESS)" project (DEM-2015-1464).

\section{CHESS project overview}

CHESS is a three-year longitudinal cohort study that is currently underway in the University College Dublin (Ireland). The project aims to: (1) evaluate the effectiveness of a $\mathrm{CH}$ platform in supporting informal caregivers of PwD at home, compared with usual care; (2) study the impact of $\mathrm{CH}$ in dementia home care, in terms of the potential improvement of the PwD and their informal caregivers' physical and mental health, and quality of life; and (3) to determine the $\mathrm{CH}$ platform's usability and user experience from the informal caregivers' perspectives. CHESS project has been reported elsewhere (GuisadoFernandez et al. 2019) and it is expected to be completed by late 2019 .

The $\mathrm{CH}$ platform works on a tablet computer (Samsung Galaxy Tab A 10.1, 2016) and it is connected to a range of PwD monitoring devices for home use, including a blood pressure (BP) monitor (Omron M6), an electronic weighing scale (Withings), and an activity and sleeping tracker
(Withings Go). From within the platform, participants have access to four main features:

- A patient educational module that provides access to information and videos from dementia experts offering advice about daily home care.

- An assessment module with daily questionnaires for the informal caregivers that gathers data separately about themselves and the PwD. In the case of PwD, data on their mood, nutrition, activity, bowel movements, and medication compliance is collected. The information is entered by the caregiver and can be remotely accessed by the healthcare team. For caregivers, surveys on their mood, energy levels, sleep quality, and anxiety levels are taken. This information will be used for research purposes only and is not accessible to the healthcare team.

- A logging module consisting of an online diary for the caregivers to keep track of events, with summary reports of changes in the PwD care plan.

- Finally, a dashboard with an overview of the PwD activity levels, sleep patterns, BP, and weight, recorded by the monitoring devices.

During the deployment, the informal caregiver has to measure the PwD's BP once a week and input the results into the dashboard section. Weight has to be measured by the informal caregiver once a month and is automatically uploaded from the scale to the health platform via Wi-Fi. Daily activity and sleeping, tracked from the activity wrist tracker, is automatically

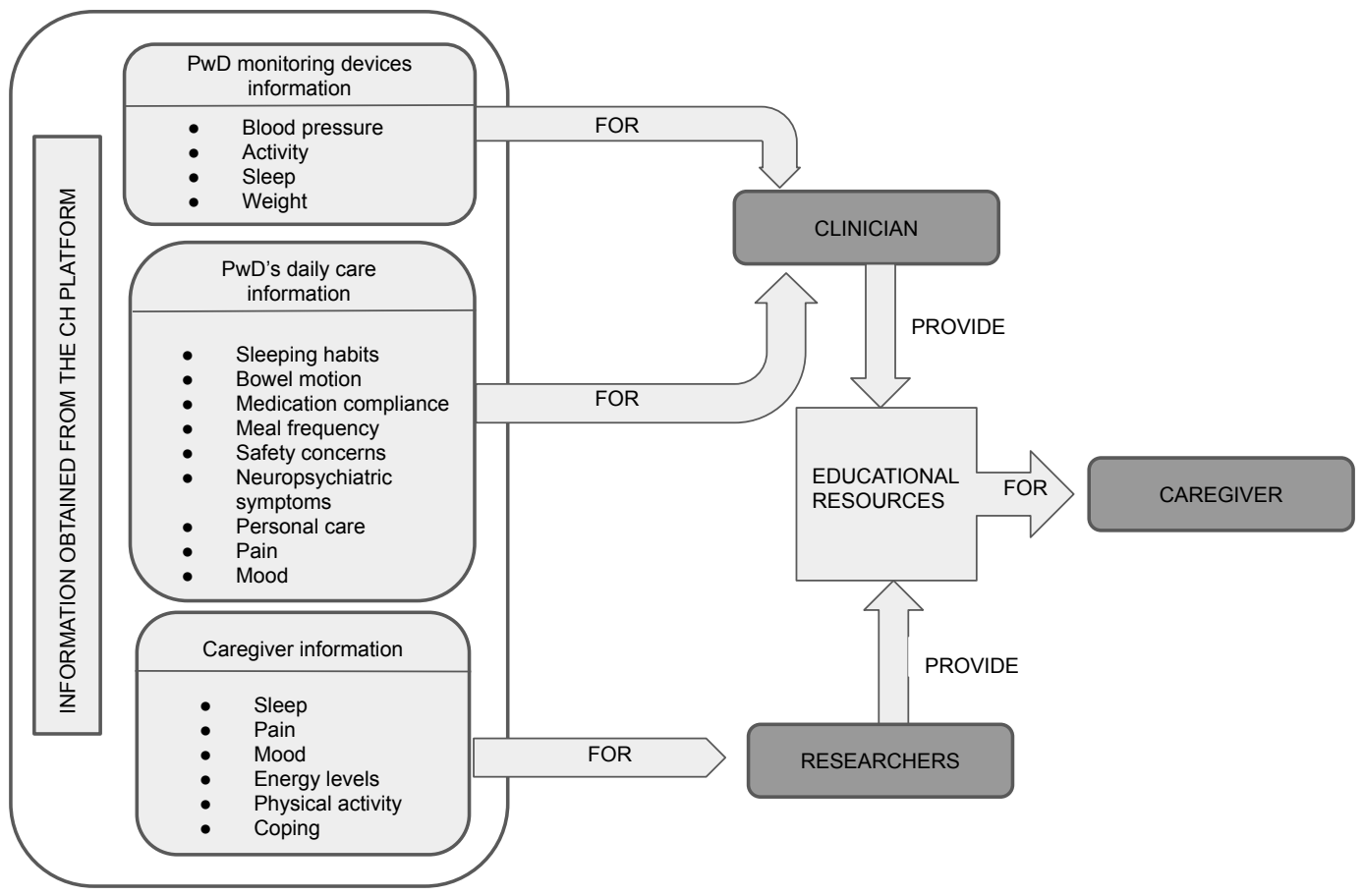

Figure 1. CHESS CH platform components.' 
uploaded via Bluetooth into the health platform. In addition, the informal caregivers are prompted through messages under each feature section name to complete the daily questionnaires on the platform, to take the weight and BP, and to have a look at the information section, which is updated with new resources regularly. The encrypted platform securely connects all the key stakeholders involved in the PwD's care (ie, informal caregiver, general practitioner, public health nurse, and hospital geriatric services). As mentioned above, the generated data is presented in the platform and made available for the informal caregivers and the health care professionals as an objective measure of the PwD's health status. Please see Figure 1 for a representation of the $\mathrm{CH}$ platform components. Screenshots of the platform interface, sections, and devices can be found in the supplementary files.

The $\mathrm{CH}$ platform was designed following a UserCentred Design (UCD) approach (Dabbs et al., 2009) which is gaining attraction in the design of health technologies (Giunti, Mylonopoulou, \& Romero, 2018). The $\mathrm{CH}$ platform is the result of an iterative process of evaluations and consultations with experts in Dementia and informal caregivers. Their input and considerations were used for developing the first prototype of the $\mathrm{CH}$ platform, which was pilot tested with informal caregivers of PwD into their homes for a short time (6 weeks). At completion, interviews were conducted with the participants to provide their feedback and user experience with the $\mathrm{CH}$ pilot platform. The knowledge acquired from the pilot study was used for making changes to the platform, in order to refine it and to align it to the PwD and caregivers' needs. The second prototype was then tested once again during a focus group with informal caregivers of PwD in our research facilities. Once more, their feedback was used for making improvements in the platform. This final resulting platform is the one under evaluation in this paper and that is currently being tested at homes by informal caregivers of PwD in the longitudinal study. An illustrative explanation of this iterative process can be seen in Figure 2.

\section{Objective}

The goal of this study is to evaluate a $\mathrm{CH}$ platform and gather feedback from informal caregivers after a long-term exposition to the platform to understand how it relates to compliance patterns.

\section{Methods \\ Study design}

We conducted a mixed-methods study to evaluate the $\mathrm{CH}$ platform described above after an extended use period in their natural living context (6 months). Informal caregivers' compliance with the $\mathrm{CH}$ platform was obtained analyzing the frequency of completion of the daily questionnaire during the deployment time.

Informal caregivers' feedback, their perceptions of platform usability, and the impact of shortterm exposure versus long-term exposure on their impressions were investigated through oneto-one semi-structured individual interviews conducted at two-time points, six months apart. These were during the initial $\mathrm{CH}$ platform deployment assessment and again at the platform removal assessment when the caregiver had the opportunity for six months of immersive use of the platform. A training session was conducted during the deployment assessment for a step-bystep explanation on how to use the $\mathrm{CH}$ platform and how to complete each task.

\section{Recruitment}

Recruitment of participants was conducted through inviting informal caregivers already involved in the CHESS project from June 2017 to December 2018 and using common inclusion criteria to that larger longitudinal project. These were: the informal caregiver must be a live-in or dedicated informal caregiver, living in the community, fluent in English (verbally and written), have adequate hearing and vision, and not be involved in another research study.

\section{Procedure}

Participants who agreed to take part in the study received a training session and were provided with a user guide containing all the information about the platform use as well as a step-by-step explanation on how to use it and how to complete each task. They were also provided with a phone number where to call in case of having any problem or needing assistance with the platform use.

A researcher (EGF) conducted the interviews following a guide which included the following topics: (1) reason for participating in the project; (2)

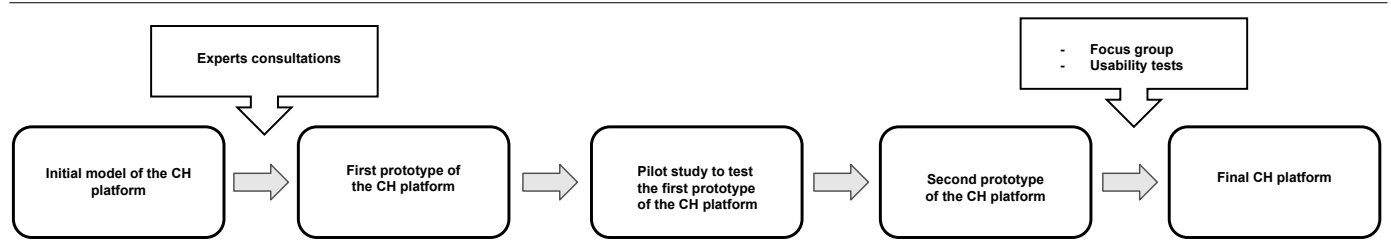

Figure 2. CHESS CH platform iterative design process. 
Table 1. Demographic characteristics of participants.

\begin{tabular}{lcc}
\hline PwD gender & & \\
\hline Male & 5 & $45.5 \%$ \\
Female & 6 & $54.5 \%$ \\
\hline PwD age & & \\
\hline Mean & 75.82 & \\
Range & $54-89$ & \\
\hline Caregiver gender & & \\
\hline Male & 4 & $36.4 \%$ \\
Female & 7 & $63.6 \%$ \\
\hline Caregiver age & & \\
\hline Mean & 66.55 & \\
Range & $48-90$ & \\
\hline Caregiver years in care & & \\
\hline Mean & 3.9 & \\
Range & $1-12$ & \\
\hline Caregiver-PwD relationship & \\
\hline \multicolumn{2}{l}{ Spouses } & \\
Children & 7 & $36.4 \%$ \\
\hline \multicolumn{2}{l}{ Type of dementia } & \\
\hline Not specified & $64.5 \%$ \\
Vascular dementia & 4 & $36.4 \%$ \\
Alzheimer's disease & 1 & $9.1 \%$ \\
\hline
\end{tabular}

platform feedback on usability, usefulness, helpfulness, expectations, suitability, and improvements; (3) caregiver impressions of PwD perceptions of the $\mathrm{CH}$ platform; and (4) caregivers' perceptions of home care technology in general. Each interview was audio-recorded and transcribed by one researcher (EGF) in the next 24 hours after the interview took place. Interviews started in June 2017 and finished by December 2018, when reached a data saturation point, as the data collection did not longer offer any new relevant data that could provide any new insights or information to our core code categories (Dworkin, 2012).

\section{Data analysis}

The transcriptions were analyzed from January to March 2019 by two researchers (EGF and LM) using content analysis, a suitable approach when analyzing technology-supported interactions (Krippendorff, 2004). It allows a qualitative analysis of the data, while at the same time allowing patterns and frequencies in the text to be measured through a systematic coding and categorization (Ryan \& Bernard, 2000; Vaismoradi, Turunen, \& Bondas, 2013). The guiding codebook used for this analysis was created following the four topics examined in the interviews as outlined above. The emergent categories were analyzed using a deductive method and organized for generating themes with Nvivo software (QSR International Pty Ltd. Version 11, 2015). The resulting themes were based on our previous Scoping Review results, as it was our leading guide for creating the interviews' questionnaire (Guisado-Fernandez et al., 2019).
Due to the lack of standard compliance measurement tools for these types of interventions, we calculated caregivers' compliance with the platform by counting up the total number of daily questionnaires completed by each caregiver and divided by the number of days they would spend with the platform. Caregivers had the platform deployed for a total of 180 days (6 months), which means that a maximally reliable caregiver would interact with the platform and complete 180 daily questionnaires during the time they were using the $\mathrm{CH}$ platform.

The compliance of any participants who withdrew earlier was calculated dividing the number of questionnaires completed till their drop out point divided by 180 (Van Het Reve, Silveira, Daniel, Casati, \& De Bruin, 2014). The results were transformed into percentages to classify them into three groups: low compliance $(0-40 \%)$, medium compliance (41-79 \%), and high compliance ( $\geq 80 \%)$.

\section{Results \\ Participants}

Eleven informal caregivers agreed to participate, completing the pre-technology deployment interview and the post technology deployment interview six months apart. From them, two PwD-informal caregiver dyads withdrew from the CHESS project, completing the post technology deployment interview at their withdrawal time. The sample was mainly composed by female informal caregivers $(63.3 \%)$, who had an average of 66.55 (SD 13.50) years of age; most of them were spouses of the PwD (63.6\%), having been a dedicated caregiver for the PwD for a mean of 3.9 years $(\min 1-\max 12)$. In terms of the PwD they were caring for, there was a reasonable balance between genders $(54.5 \%$ female versus $45.5 \%$ male), and the PwD had an average of 75.82 (SD 10.78) years of age. The majority of the PwD had a non-specified type of Dementia (54.5\%); the second most common type was Vascular Dementia (36.4\%) followed by Alzheimer's Disease (9.1\%) (Table 1).

\section{Participants' compliance with the $\mathrm{CH}$ platform} Caregivers' compliance with the $\mathrm{CH}$ platform was quite similar between all participants. Most of the caregivers had low compliance with the platform (72.72\%); just $2 / 11(18.18 \%)$, caregivers had medium compliance and only $1 / 11$ (9.09\%) had high compliance (Table 2).

\section{Participants' feedback and time-exposure effect} A few themes emerged from the interviews. At the technology deployment, our participants created a conceptualization of what the $\mathrm{CH}$ platform could provide them in terms of their own support and were quite optimistic about the benefits that it could bring into their daily caring duties. After the 
Table 2. Participants' compliance to the platform.

\begin{tabular}{lccc}
\hline $\begin{array}{l}\text { Participant } \\
\text { ID }\end{array}$ & $\begin{array}{c}\text { Number of daily } \\
\text { questionnaires completed }\end{array}$ & \% of compliance & $\begin{array}{c}\text { Group } \\
\text { (Low/Medium/High) }\end{array}$ \\
\hline C1 & 102 & 56.6 & Medium \\
C2 & 40 & 22.2 & Low \\
C3 & 166 & 92.2 & High \\
C4 & 67 & 37.2 & Low \\
C5 & 34 & 18.8 & Low \\
C6 & 15 & 8.3 & Low \\
C7 & 66 & 36.6 & Low \\
C8 & 117 & 65.0 & Medium \\
C9 & 23 & 12.7 & Low \\
C10 & 9 & 5.0 & Low \\
C11 & 41 & 22.7 & Low \\
\hline
\end{tabular}

them in their daily care duties and would allow them to monitor the PwD's health ("It will help me to keep on top of things"). After deployment, most of the participants agreed that the platform did not provide such health monitoring support, as they thought that the health parameters monitored and questionnaires asked were not relevant for their caring needs ("I didn't actually find it many benefits for me really"), except for the sleeping tracking and BP monitoring. These devices were seen as a facilitator for providing better communication with the clinicians about any health issues in the PwD ("When he says to me I never slept a wink. So, I said to look back on that and tell me. But you slept nine hours. He told me I didn't sleep"; "I would like to keep the blood pressure one because of the fact that sometimes Lisa has these drops, I'm able to tell her doctor what her blood pressure is regularly and then what I have noticed").

\section{Self-efficacy facilitator}

In the beginning, some participants saw their participation as a benefit for themselves and the PwD, expecting that the platform would provide them with more independence and more knowledge about what to expect as the disease progresses ("Yes, I think it's going to be greatly beneficial for giving me more freedom or independence in terms of keeping an eye on her health"). This idea was not expressed during the after deployment interviews.

\section{Perceived usefulness for care facilitation}

Our participants considered from the beginning that the dashboard section was the most helpful piece in the platform for facilitating their daily caring work, most of all the sleeping and the activity tracker. The reason for that was that, as some of the PwD were struggling with their sleeping patterns and had a sedentary life, the informal caregivers hoped that having it tracked could help them to show this to the doctors and take some measures to solve it "He goes up at 7 pm and he doesn't get up until around 12 pm next day, [...] so I will monitor that and go to his doctor"). Just one participant mentioned that at first, couldn't think of any usefulness in the daily questionnaire for their daily duties ("The questionnaire that you have to fill in every day, I don't think it's going to help me in any way"). Another two thought the same about the weight measurement. For the rest of the participants, all the features seemed to have a purpose and found 
them of some value. After deployment, while the dashboard was still considered the most useful feature, the rest of the features had totally been neglected, most of all the daily questionnaires and the information resources. They were not considered useful by most of the participants, having found them monotonous to complete and too repetitive ("...they are every day the same, nothing changed, I got careless about it!").

\section{Usability}

In general, the whole system was found easy to use. Only two participants considered in the beginning that their lack of technological skills and education would make it a bit difficult to use ("I'm not good at technology..."). However, these two same participants mentioned after the deployment that they found the platform easy to use, and that their lack of digital literacy did not influence them in the platform utilization ("It was easy to use for what I could cope with").

\section{Mismatched expectations and needs}

As mentioned above, at the beginning most of the participants seemed to be quite happy with their participation and saw some kind of benefit for them or the PwD derived from it. After removing the devices, we asked the participants if, given the opportunity, they would like to keep using the $\mathrm{CH}$ platform at home; 7/11 participants recognized that they would not like to keep using it, as the platform did not meet their initial impressions and did not help them with what they anticipated ("I'm not very sure what benefits for us..."). Most of the participants commented that, because of the way the project was built and deployed, it was too medicalized, and that the health parameters monitored for the PwD were not the information that they needed for providing good care ("Why would I need to know how many steps Linda takes? And she sleeps so well that there's no need... "). Only 4/11 participants kept thinking of it in a positive way, confirming the impressions and thoughts verbalised in the first interview and supporting their initial insights ("You know if she wasn't doing anything, like walking or sleeping enough, I wouldn't have known that otherwise. If I got on the phone my mother would have said something different").

\section{Familiarization}

When asked about any initial thoughts about how could the platform be improved for making it more helpful for their caring tasks, most of the informal caregivers commented that it was too early to think of any ("It's too early to say..."). They all made clear that they need more time to learn to navigate through it and get familiarised with its functions before finding out how it could be improved.
Need for platform personalization

If at the beginning every feature seemed to have a purpose for most of all the participants, after the deployment, this was not seen in the same way. The participants mentioned that the daily questionnaires were not applicable to the PwD and their caring situation, not being adapted to each individual's disease stage and needs ("The questions mostly adhere to a person with a little activity or with other difficulties [...], they are not relevant to me and my mother's disease stage"), and without an appropriate frequency ( "...even once a month would be enough..."). All participants agreed on the need for changing the frequency of the questionnaire and adapting the content to each individual ("Everybody has their own queries"). Something similar happened to the educational module; just two participants affirmed having had a look at them and considered it revealing ("I have used all of them, they are really good, and I got my kids to watch them as well"'). The rest of the participants replied that they did not have a look at it, either because they forgot about its existence ("Where is this now? On the platform?"), were busy with other caring issues ("The piece that gives you information...l didn't use that. To be honest, with all that's been going on I just..."), the information provided was not considered necessary, as already been aware of the condition and resources available ("Because as you know l've done a couple of online courses and I didn't think it could add anything to that. I suppose I should have looked at it to see if it could teach me anymore"; "I thought it was good. It ended into very late stages of Dementia so it didn't really apply anymore, but it could be interesting for someone else"), or because they did not found it relevant to the disease stage ("It doesn't equate to what John is at the moment...").

\section{Room to improve}

At the deployment, most of the participants mentioned that at first, they couldn't think of any platform changes ("I can't think offhand of anything, I'll know more in a few months"). This same question had a totally different answer when asked after the deployment. Apart from the recommendations about the questionnaires and the education section, some participants recommended the addition of a heart rate monitoring and a GPS location. They also recommended making the tracking device to look more like a piece of jewelry and not as a clinical device in order to avoid any misinterpretation from the PwD due to their cognitive decline. Also, the participants showed interest in having added to the $\mathrm{CH}$ platform some type of social network to contact other informal caregivers, update the content with community meetings with other peers, and to add messages or beeping reminders to the tasks that have to be completed instead 
of just a pop-up message.

\section{Technology burden}

Despite the platform was not considered in the beginning that it could bring any added burden for our busy participants, after the deployment it was considered burdensome in some ways. Two of the participants had to take the PwD to their HCP because the BP monitor was constantly giving a BP measurement higher than what was usual for the PwD, making them worry ("I took the blood pressure a couple of times in the beginning and it was right off the scale...we got him over at the local doctor who said it was fine... it kind of put us off that a bit so I just stopped using $i t^{\prime \prime}$ ). Another participant could not take the PwD $\mathrm{BP}$ measured as the measurements were giving a constant error with the PwD, but worked properly when measuring it in other family members.

\section{Technology resistance}

Just one participant considered in the beginning that the PwD would be resistant to wear the activity and sleeping tracker, just because of the own disease nature making her forget what that was ("I suppose the thing is she won't really know, but it's just the wearing of the thing... but once it's on there shouldn't be any issues. And it's a little bit difficult to take off so hopefully, that will help" $p^{\prime \prime}$. Furthermore, one of the participants that withdrew before finishing the deployment time commented that the reason for leaving was that the PwD did not want to be monitored, as did not want the informal caregiver to see how sedentary she was ("Granny has refused to, she doesn't like the fact that you can tell how long she has slept or how little she has walked. That is the only reason she's not wearing it"). The rest of the participants mentioned that the PwD did not offer any resistance to the platform use and monitoring, neither at the on-boarding or at the leaving interview.

This can be summarized in a few lessons:

- UCD is key to $\mathrm{CH}$ success. However, applying UCD does not mean that your technology is going to be fruitful. Before starting the design of a $\mathrm{CH}$ technology solution, we need to fully understand the intended users' needs. In the case of Dementia, we need to analyze the disease demands and their caring needs at home. This can only be achieved including them from the early beginning of the design process. We also need to make them tell us how they see that technology could facilitate those caring needs. In our case, despite having involved informal caregivers from the beginning of the process, our $\mathrm{CH}$ platform was not a success. Probably, the main reason for that was our lack of understanding of their true needs. Our participants also preferred a less medicalized platform and more social support oriented. Adding social media components such as a communication tool with other informal caregivers or social meetings.

- The well known saying 'one size does not fit all' has totally been proved here. Despite PwD may have symptoms and care needs in common, each individual is different and therefore their caring demands are. Also, each individual may have some fluctuation in their symptoms which may vary in length. Therefore, $\mathrm{CH}$ needs to be adapted and personalized to each individuals needs and stage.

- People need time to get familiarised with a piece of technology before deciding if it really fits into their needs. Hence, being exposed to technology during an extended period can help to get a true insight into the technical features and what it can offer for the user.

\section{Discussion \\ Principal findings}

Our study is unique in the way we have organized and presented our findings, combining quantitative data and qualitative content analysis in a descriptive narrative way. This allowed us to report the findings outlined in the caregivers' interviews in comparison with the quantitative data gathered from their daily log, giving us a richer more realistic picture of what happened during the deployment time (Ben Mortenson et al., 2018; Kernisan, Sudore, \& Knight, 2010; Lundberg, 2014; Mortenson et al., 2018). Furthermore, most of the studies published at the moment principally report on positive findings (Boots et al., 2013), however in our case, we are reporting all pros and cons expressed by our participants, as we consider their negative feedback not as a failure but as constructive criticism to keep improving our work. Despite our iterative UCD process involving Dementia and $\mathrm{CH}$ experts, as well as PwD informal caregivers, the system seems to do not properly fit into their daily caring needs and does not match with their initial impressions. This supports literature highlighting the importance of involving PwD and their informal caregivers in the design process from the very beginning for enhancing usability and technology acceptance (Archer, Keshavjee, Demers, \& Lee, 2014; Wan, Byrne, O'Grady, \& O'Hare, 2015).

There was a clear change in our caregivers' opinions and points of view about the $\mathrm{CH}$ platform when comparing the short-term and the longterm exposure feedback. It seems that the caregivers' initial impressions about what the $\mathrm{CH}$ platform features could provide them for the PwD home care did not correspond with what they found following the long-term exposure. During the interviews, the informal caregivers made clear their various reasons for not complying with the $\mathrm{CH}$ platform. Initially, the caregivers 
saw the $\mathrm{CH}$ platform as a benefit for the PwD health monitoring and as an opportunity to empower themselves in their caring duties. After the deployment, they essentially conceived the platform for research's benefit. Hence, the unmet informal caregivers' needs and expectations played an important part in the lack of compliance and negative long-term feedback.

Another frequently mentioned cause for not compliance was the lack of personalization and tailoring of the content of the platform. The informal caregivers felt that the questions asked were too clinical and not relevant to the PwD stage and their care needs and that a customization of them based on each individual's situation and disease stage would be more appropriate ("Well, they weren't practical probably. I know you have to have a set number of questions but probably weren't relevant in my mother's case"). The informal caregivers recommended customizing the resources section content as well, as some of them pointed out that the content was either too basic for them or focused into Dementia stages that didn't apply to them ("It ended into very late stages of Dementia so...it didn't really apply anymore"). These suggestions made us consider that negotiation about shared goals, personalization of monitoring parameters, and clarity in expectations are important to set out the prior deployment. This was a research prototype and thus content was relatively generic to cater to all. For real-world deployment, we do need tailoring of the right information and right assessments for the right person, following a needs-based assessment with the caregiver and PwD as key decision-makers.

Despite having conducted a training session and providing the informal caregivers with a user guide and a contact phone, many of them pointed out that this was not sufficient for understanding the $\mathrm{CH}$ platform functioning and how they could use all that data and information gathered for their and the PwD's benefit. They argue that having been provided with more training at the beginning would have been beneficial for learning how to use the platform and getting familiarized with it, as well as understanding how the $\mathrm{CH}$ platform could empower them in their caring duties. We also consider that providing more opportunities for this training could have helped the caregivers to gain a clear insight into the $\mathrm{CH}$ platform's aim and possibilities for Dementia home care, how they could benefit, and probably could have avoided those expectations that were not realized.

Despite this negative feedback in the long-term, the informal caregivers were still quite optimistic about the promising benefits of using $\mathrm{CH}$ tech- nologies for Dementia home care and were motivated to take part in future Dementia research. Some of them had even started to be engaged with new technologies during the deployment time that they had never used before: one participant got a smartphone from her kids because of starting using the tablet ("My daughter-in-law gave me another phone the other day to use, a modern one"), another got an iPad and a Fitbit tracker, and another one bought an Amazon Alexa that used for playing music for entertaining the PwD. This also denotes a generational difference in our population, between the younger and technology-educated ones and the older and non-technology educated.

Having said this, our impression is that with this $\mathrm{CH}$ platform prototype we have not provided our informal caregivers with the things they may need for their daily caring life. Furthermore, what we provided was probably not given in the appropriate way, and without a proper explanation of what using $\mathrm{CH}$ could mean for Dementia home care. We should have made more emphasis in that all the information collected and showed in the $\mathrm{CH}$ platform creates a pathway for the informal caregiver to actively and meaningfully use it to support home care for the PwD. It could be possible that we did not provide them at the deployment phase with the appropriate information on what all these data means or what they could do with it, and what an empowered informal caregiver can do for a PwD and for themselves. From their insights, we have learned that they are willing for a more person-centered and that more clarity from the beginning of what technology can offer can help them to properly set out their expectations from the start.

\section{Comparison with prior work}

Our major findings are consistent with previous similar studies involving feedback from informal caregivers of PwD. Dementia informal caregivers are willing to use technology for the home care (Evans, Harris, \& Kuppuswamy, 2011; Mahoney \& Mahoney, 2010), but researchers have to provide solutions that truly cover their needs and expectations (Cristancho-Lacroix et al., 2015; Faucounau, Wu, Boulay, Maestrutti, \& Rigaud, 2009; Patterson T, McClean S, Langdon PM, Zhang S, Nugent C, 2014), are personalized (Alm et al., 2004; Boyd et al., 2014; Mitseva et al., 2012), provide relevant and reliable information (Brankaert, Snaphaan, \& den Ouden, 2014), and are easy to use (Orpwood, Gibbs, Adlam, Faulkner, \& Meegahawatte, 2005; Sugihara, Fujinami, Phaal, \& Ikawa, 2012; Williams et al., 2016). However, in previously published studies on usability and users feedback, there are some topics frequently mentioned that we were expecting to get from our caregivers' feedback as 
well, but did not, like the possibility that the platform really provided them with more independence and freedom (Begum et al., 2013).

\section{Limitations}

There are some limitations to consider when interpreting our results. The first is that despite the $\mathrm{CH}$ platform has been designed to connect all the stakeholders involved in PwD's care, our study is only focused on the informal caregivers' perspectives. Hence, some important insights may be added when investigating other stakeholders' opinions. We also have to consider as a limitation that our informal caregivers had many different backgrounds and a wide difference between their age, which can lead to a gap between two generations: the technology educated and the non-technology educated, being these last ones the most predominant in our group. Another limitation is that we can not give a "rule of thumb" for our results and extrapolate them to other similar studies conducted in the field, as we have to consider our outcomes and findings

\section{Acknowledgements}

The authors EG-F and GG gratefully acknowledge a grant (\#676201) for the Connected Health Early-Stage Researcher Support System (CHESS ITN) from the Horizon 2020 Framework Programme of the European Commission.

The author LMM gratefully acknowledges a grant (DEM2015-1464) from the Health Research Board Ireland (HRB).

The authors declare no conflicts of interest.

\section{References}

Alm, N., Astell, A., Ellis, M., Dye, R., Gowans, G., \& Campbell, J. (2004). A cognitive prosthesis and communication support for people with dementia. Neuropsychological Rehabilitation, 14(1-2), 117-134. https://doi.org/10.1080/09602010343000147

Archer, N., Keshavjee, K., Demers, C., \& Lee, R. (2014). Online self-management interventions for chronically ill patients: Cognitive impairment and technology issues. International Journal of Medical Informatics, 83(4), 264-272. https://doi.org/10.1016/j. ijmedinf.2014.01.005

Cahill, S., Macijauskiene, J., Nygård, A. M., Faulkner, J. P., \& Hagen, I. (2007). Technology in dementia care. Technology and Disability, 19(2, 3), 55-60.

Begum, M., Wang, R., Huq, R., \& Mihailidis, A. (2013, June). Performance of daily activities by older adults with dementia: The role of an assistive robot. In 2013 IEEE 13th International Conference on Rehabilitation Robotics (ICORR) (pp. 1-8). IEEE. https://doi.org/10.1109/ICORR.2013.6650405

Ben Mortenson, W., Demers, L., Fuhrer, M. J., Jutai, J. W., Bilkey, J., Plante, M., \& DeRuyter, F. (2018). Effects of a caregiver-inclusive assistive technology intervention: a randomized controlled trial. BMC Geriatrics, 18(1), 97. https://doi.org/10.1186/ s12877-018-0783-6 within this specific project features, such as its context, timing, population, and offered content.

\section{Conclusions}

$\mathrm{CH}$ is a novel concept in practice which does offer the potential for PwD's home monitoring and informal caregiver support. Most contemporary $\mathrm{CH}$ studies are not conducted in real-life settings and without enough duration in time. Consequently, this is not providing accurate insights into the factual informal caregivers' interaction with these technologies and their suitability for their caring needs and support. With this study, we have recognized the importance of studying how informal caregivers' are engaging with $\mathrm{CH}$ for home care in their own environments and for enough duration of time. We have also highlighted that, despite the fact of applying UCD, the result may not always be satisfactory for the user. For these reasons, more longitudinal research on $\mathrm{PwD}$ and their informal caregivers, $\mathrm{CH}$ technologies adoption need to be conducted.

Bharucha, A. J., Anand, V., Forlizzi, J., Dew, M. A., Reynolds, C. F., Stevens, S., \& Wactlar, H. (2009). Intelligent assistive technology applications to dementia care: Current capabilities, limitations, and future challenges. American Journal of Geriatric Psychiatry, 17(2), 88-104. https://doi.org/10.1097/ JGP.0b013e318187dde5

Boletsis, C., McCallum, S., \& Landmark, B. F. (2015, August). The use of smartwatches for health monitoring in home-based dementia care. In International Conference on Human Aspects of IT for the Aged Population (pp. 15-26). Springer, Cham. https://doi. org/10.1007/978-3-319-20913-5_2

Boots, L. M. M., Van Knippenberg, R. J. M., Kempen, G. I. J. M., Verhey, F. R. J., de Vugt, M. E., Van Knippenberg, R. J. M., \& Verhey, F. R. J. (2013). A systematic review of Internet-based supportive interventions for caregivers of patients with dementia. International Journal of Geriatric Psychiatry, 29(4), 331-344. https://doi.org/10.1002/gps.4016

Bossen, A. L., Kim, H., Williams, K. N., Steinhoff, A. E., \& Strieker, M. (2015). Emerging roles for telemedicine and smart technologies in dementia care. Smart Homecare Technology and Telehealth, 3, 49-57. https://doi.org/10.2147/SHTT.S59500.

Boyd, K., Nugent, C., Donnelly, M., Bond, R., Sterritt, R., \& Hartin, P. (2014, August). An investigation into the usability of the STAR training and re-skilling website for carers of persons with dementia. In 2014 36th Annual International Conference of the IEEE Engineering in Medicine and Biology Society (pp. 4139-4142). IEEE. https://doi.org/10.1109/ EMBC.2014.6944535

Brankaert, R., Snaphaan, L., \& den Ouden, E. (2014, December). Stay in touch: an in context evaluation of a smartphone interface designed for people with dementia. In International Workshop on Ambient 
Assisted Living (pp. 288-295). Springer, Cham. https://doi.org/10.1007/978-3-319-13105-4_42

Brooker, D. (2004). What is person-centred care in dementia? Reviews in Clinical Gerontology, 13(3), 215-222. https://doi.org/10.1017/ S095925980400108X

Brooker, D., \& Latham, I. (2015). Person-centred dementia care: making services better with the VIPS framework. Jessica Kingsley Publishers.

Brown, M., Tsai, A., Baurley, S., Koppe, T., Lawson, G., Martin, J., ... \& Arunachalam, U. (2014, June). Using cultural probes to inform the design of assistive technologies. In International Conference on Human-Computer Interaction (pp. 3546). Springer, Cham. https://doi.org/10.13140/ RG.2.1.3976.2965

Caulfield, B. M., \& Donnelly, S. C. (2013). What is Connected Health and why will it change your practice? QJM: An International Journal of Medicine, 106(8), 703-707. https://doi.org/10.1093/qjmed/hct114

Chouvarda, I. G., Goulis, D. G., Lambrinoudaki, I., \& Maglaveras, N. (2015). Connected health and integrated care: Toward new models for chronic disease management. Maturitas, 82(1), 22-27. https:// doi.org/10.1016/j.maturitas.2015.03.015

Cristancho-Lacroix, V., Wrobel, J., Cantegreil-Kallen, I., Dub, T., Rouquette, A., \& Rigaud, A. S. (2015). A web-based psychoeducational program for informal caregivers of patients with Alzheimer's disease: A pilot randomized controlled trial. Journal of Medical Internet Research, 17(5). https://doi. org/10.2196/jmir.3717

Dabbs, A. D. V., Myers, B. A., Curry, K. R. M., Dunbarjacob, J., Hawkins, R. P., Begey, A., \& Dew, M. A. (2009). User-centered design and interactive health technologies for patients. Computers, Informatics, Nursing : CIN, 27(3), 175. https://doi.org/10.1097/ NCN.0b013e31819f7c7c.

Darkins, A., Ryan, P., Kobb, R., Foster, L., Edmonson, E., Wakefield, B., \& Lancaster, A. E. (2008). Care Coordination/Home Telehealth: The Systematic Implementation of Health Informatics, Home Telehealth, and Disease Management to Support the Care of Veteran Patients with Chronic Conditions. Telemedicine and E-Health, 14(10), 1118-1126. https://doi.org/10.1089/tmj.2008.0021

Donelan, K., Hill, C. A., Hoffman, C., Scoles, K., Feldman, P. H., Levine, C., \& Gould, D. (2002). Challenged to care: Informal caregivers in a changing health system. Health Affairs, 21(4), 222-231. https://doi.org/10.1377/hlthaff.21.4.222

Dworkin, S. L. (2012). Sample size policy for qualitative studies using in-depth interviews.

Epp, T. D. (2003). Person-centred dementia care: A vision to be refined. The Canadian Alzheimer Disease Review, 5(3), 14-19.

Evans, N., Harris, N., \& Kuppuswamy, A. (2011). A smarter future: Technology to enhance an independent lifestyle for our future selves. International Journal of Therapy and Rehabilitation, 18(12), 694699. https://doi.org/10.12968/ijtr.2011.18.12.694

Faucounau, V., Wu, Y.-H., Boulay, M., Maestrutti, M., \& Rigaud, A.S. (2009). Caregivers' requirements for in-home robotic agent for supporting community- living elderly subjects with cognitive impairment. Technology and Health Care, 17(1), 33-40. https:// doi.org/10.3233/THC-2009-0537

Feeney Mahoney, D., Coon, D. W., \& Lozano, C. (2016). Latino/Hispanic Alzheimer's caregivers experiencing dementia-related dressing issues: corroboration of the Preservation of Self model and reactions to a "smart dresser" computer-based dressing aid. Digital Health, 2, 2055207616677129. https://doi. org/10.1177/2055207616677129

Giunti, G., Mylonopoulou, V., \& Romero, O. R. (2018). More stamina, a gamified mhealth solution for persons with multiple sclerosis: research through design. JMIR mHealth and uHealth, 6(3), e51. https:// doi.org/10.2196/mhealth.9437

Godwin, K. M., Mills, W. L., Anderson, J. A., \& Kunik, M. E. (2013). Technology-driven interventions for caregivers of persons with dementia: A systematic review. American Journal of Alzheimer's Disease and Other Dementias, 28(3), 216-222. http://doi. org/10.1177/1533317513481091

Guisado-Fernandez, E., Caulfield, B., Silva, P.A., Mackey, L., Singleton, D., Leahy, D., Dossot, S., Power, D., O'Shea, D., Blake, C. (2019). Development of a Caregivers' Support Platform (Connected Health Sustaining Home Stay in Dementia): Protocol for a Longitudinal Observational Mixed Methods Study. JMIR Research Protocols, 8(8):e13280. https://doi. org/10.2196/13280

Guisado-Fernández, E., Giunti, G., Mackey, L. M., Blake, C., \& Caulfield, B. M. (2019). Factors Influencing the Adoption of Smart Health Technologies for People With Dementia and Their Informal Caregivers: Scoping Review and Design Framework. JMIR Aging, 2(1), e12192. https://doi.org/10.2196/12192

Hattink, B., Droes, R. M., Sikkes, S., Oostra, E., \& Lemstra, A. W. (2016). Evaluation of the Digital Alzheimer Center: testing usability and usefulness of an online portal for patients with dementia and their carers. JMIR research protocols, 5(3). https://doi. org/10.2196/resprot.5040

Hwang, A. S., Truong, K. N., \& Mihailidis, A. (2012, May). Using participatory design to determine the needs of informal caregivers for smart home user interfaces. In 2012 6th International Conference on Pervasive Computing Technologies for Healthcare (PervasiveHealth) and Workshops (pp. 41-48). IEEE. https://doi.org/10.4108/icst.pervasivehealth.2012.248671

Johnson, C. M., Johnson, T. R., \& Zhang, J. (2005). A user-centered framework for redesigning health care interfaces. Journal of Biomedical Informatics, 38(1), 75-87. https://doi.org/https://doi.org/10.1016/j. jbi.2004.11.005

Kernisan, L. P., Sudore, R. L., \& Knight, S. J. (2010). Information-Seeking at a Caregiving Website: A Qualitative Analysis. Journal of Medical Internet Research, 12(3). https://doi.org/10.2196/jmir.1548

Kitwood, T. (1995). Building up the mosaic of good practice. Journal of Dementia Care, 3(5), 12-13.

Kort, H. S. M. M., \& Van Hoof, J. (2014). Design of a website for home modifications for older persons with dementia. Technology and Disability, 26(1), 1-10. https://doi.org/10.3233/TAD-140399 
Krippendorff, K. (2004). Content analysis: an introduction to its methodology. SAGE publications.

Lewis, M. L., Hobday, J. V, \& Hepburn, K. W. (2010). Internet-based program for dementia caregivers. American Journal of Alzheimer's Disease and Other Dementias, 25(8), 674-679. https://doi. org/10.1177/1533317510385812

Lopes, P., Pino, M., Carletti, G., Hamidi, S., Legué, S., Kerhervé, H., ... \& Rigaud, A. S. (2016). Co-Conception Process of an Innovative Assistive Device to Track and Find Misplaced Everyday Objects for Older Adults with Cognitive Impairment: The TROUVE Project. IRBM, 37(2), 52-57. https://doi. org/10.1016/j.irbm.2016.02.004

Lundberg, S. (2014). The results from a two-year case study of an information and communication technology support system for family caregivers. Disability and Rehabilitation: Assistive Technology, 9(4), 353-358. https://doi.org/10.3109/17483107.2 013.814170

Mahoney, E. L., \& Mahoney, D. F. (2010). Acceptance of wearable technology by people with alzheimer's disease: Issues and accommodations. American Journal of Alzheimer's Disease and Other Dementias, 25(6), 527-531. https://doi. org/10.1177/1533317510376944

Mitseva, A., Peterson, C. B., Karamberi, C., Oikonomou, L. C., Ballis, A. V., Giannakakos, C., \& Dafoulas, G. E. (2012). Gerontechnology: Providing a helping hand when caring for cognitively impaired older adults-intermediate results from a controlled study on the satisfaction and acceptance of informal caregivers. Current Gerontology and Geriatrics Research, 2012, 1-19. https://doi. org/10.1155/2012/401705

Mortenson, W. Ben, Pysklywec, A., Fuhrer, M. J., Jutai, J. W., Plante, M., \& Demers, L. (2018). Caregivers' experiences with the selection and use of assistive technology. Disability and Rehabilitation: Assistive Technology, 13(6), 562-567. https://doi.org/10.108 0/17483107.2017.1353652

Morton, I. (1999). Person-centred approaches to dementia care. Winslow Press.

Orpwood, R., Gibbs, C., Adlam, T., Faulkner, R., \& Meegahawatte, D. (2005). The design of smart homes for people with dementia - User-interface aspects. Universal Access in the Information Society, 4(2), 156-164. https://doi.org/10.1007/s10209005-0120-7

Patterson, T., McClean, S., Langdon, P. M., Zhang, S., Nugent, C., \& Cleland, I. (2014, August). A knowledge-driven approach to predicting technology adoption among persons with dementia. In 2014 36th Annual International Conference of the IEEE Engineering in Medicine and Biology Society (pp. 5928-5931). IEEE. https://doi.org/10.1109/ EMBC.2014.6944978

Pot, A. M., Blom, M. M., \& Willemse, B. M. (2015). Acceptability of a guided self-help Internet intervention for family caregivers: Mastery over dementia. International Psychogeriatrics, 27(8), 1343-1354.: https://doi.org/10.1017/S1041610215000034

Ryan, G. W., Bernard, H. R., Denzin, N., \& Lincoln, Y. (2000). Handbook of qualitative research. Handbook of qualitative research. https://doi. org/10.1016/S0031-9406(05)61288-6

Span, M., Smits, C., Groen-van de Ven, L. M., Cremers, A., Jukema, J., Vernooij-Dassen, M., \& Hettinga, M. (2014). An interactive web tool to facilitate shared decision making in dementia: design issues perceived by caregivers and patients. Int. J. Adv. Life Sci, 6, 107-121.

Sugihara, T., Fujinami, T., Phaal, R., \& Ikawa, Y. (2012, July). Gaps between assistive technologies and dementia care. In 2012 Proceedings of PICMET'12: Technology Management for Emerging Technologies (pp. 3067-3072). IEEE.

Torkamani, M., McDonald, L., Aguayo, I. S., Kanios, C., Katsanou, M. N., Madeley, L., ... \& Jahanshahi, M. (2014). A randomized controlled pilot study to evaluate a technology platform for the assisted living of people with dementia and their carers. Journal of Alzheimer's disease, 41(2), 515-523. https:// doi.org/10.3233/JAD-132156

Vaismoradi, M., Turunen, H., \& Bondas, T. (2013). Content analysis and thematic analysis: Implications for conducting a qualitative descriptive study. Nursing and Health Sciences, 15(3), 398-405. https://doi. org/10.1111/nhs.12048

Van Het Reve, E., Silveira, P., Daniel, F., Casati, F., \& De Bruin, E. D. (2014). Tablet-based strength-balance training to motivate and improve adherence to exercise in independently living older people: part 2 of a phase II preclinical exploratory trial. Journal of medical internet research, 16(6). https://doi. org/10.2196/jmir.3055

Wan, J., Byrne, C. A., O'Grady, M. J., \& O'Hare, G. M. P. P. (2015). Managing Wandering Risk in People With Dementia. IEEE Transactions on Human-Machine Systems, 45(6), 819-823. https://doi.org/10.1109/ THMS.2015.2453421

Williams, K., Pennathur, P., Bossen, A., \& Gloeckner, A. (2015). Adapting telemonitoring technology use for older adults: a pilot study. Research in gerontological nursing, 9(1), 17-23. https://doi. org/10.3928/19404921-20150522-01

World Health Organization. (2018). Towards a dementia plan: a WHO guide. 